\title{
A photoswitchable antimetabolite for targeted photoactivated chemotherapy
}

\author{
Carlo Matera ${ }^{\dagger, \S}$, Alexandre Gomila-Juaneda ${ }^{\dagger, \S,}$, Núria Camarero ${ }^{\dagger, \S, \$}$, Michela Libergoli ${ }^{\dagger}$, Concepció So- \\ ler $^{\#}$, and Pau Gorostiza ${ }^{\dagger, \$, \text {,* }}$ \\ ${ }^{\dagger}$ Institute for Bioengineering of Catalonia (IBEC), Barcelona Institute for Science and Technology (BIST) \\ ${ }^{\S}$ Biomedical Research Networking center in Bioengineering, Biomaterials and Nanomedicine (CIBER-BBN)

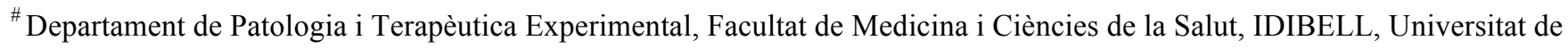 \\ Barcelona \\ "Catalan Institution for Research and Advanced Studies (ICREA)
}

photopharmacology, photodynamic therapy, antiproliferative, arthritis, psoriasis, nanomedicine

\begin{abstract}
The efficacy and tolerability of systemically administered anticancer agents are limited by their off-target effects. Precise spatiotemporal control over their cytotoxic activity would allow improving chemotherapy treatments, and light-regulated drugs are well suited to this purpose. We have developed phototrexate, the first photoswitchable inhibitor of the human dihydrofolate reductase (DHFR), as a photochromic analog of methotrexate, a widely prescribed chemotherapeutic drug to treat cancer and psoriasis. Quantification of the light-regulated DHFR enzymatic activity, cell proliferation, and in vivo effects in zebrafish show that phototrexate behaves as a potent antifolate in its photoactivated cis configuration, and that it is nearly inactive in its darkrelaxed trans form. Thus, phototrexate constitutes a proof-of-concept to design light-regulated cytotoxic small molecules, and a step forward to develop targeted anticancer photochemotherapies with localized efficacy and reduced adverse effects.
\end{abstract}

\section{INTRODUCTION}

Although the neoplastic process has been recognized for centuries, the treatment and cure of cancer still represent one of the main challenges in medicine. ${ }^{1}$ Chemotherapy - the use of cytotoxic agents to kill the rapidly proliferating cells present in tumors - is one of the main actors in this fight, together with radiation therapy, hormone therapy and surgery. ${ }^{2}$ However, the efficacy, tolerability and acceptance of cancer chemotherapy are in many cases dramatically limited by low therapeutic indices, non-specific targeting, and off-target toxicity of the drugs used, which is intrinsic to their antiproliferative effects. ${ }^{3}$ Alternative strategies to overcome these drawbacks have emerged over the last years.

One approach relies on the design of pharmacological agents interfering with targets that are overexpressed in tumor cells, i.e. growth factors, signaling molecules, cell-cycle proteins, and modulators of apoptosis and angiogenesis. ${ }^{1,4,5}$ However, despite remarkable achievements obtained recently, the development of such targeted cancer therapies is hampered by the identification of tumor biomarkers, the selection of patientpersonalized treatments, and high costs. ${ }^{6,7}$ Another strategy to reduce off-target toxicity in cancer treatments relies on the design of engineered delivery systems (e.g., nanoparticles, liposomes, micelles) that can selectively release the chemotherapeutic at the tumor site. The increased availability of versatile materials and recent advances in the field of nanotechnology are certainly boosting this field, but yet the clinical translation of such drug carriers has been very limited so far. ${ }^{3}$

A third approach to improve cancer therapies is to activate the drugs exclusively at their desired place of action. ${ }^{8}$ In fact, in cancer types and stages that would benefit from a highly localized treatment, a precise spatiotemporal control over the activity of a cytotoxic agent would allow reducing the concentration of active compound outside the targeted neoplasm region, and would permit the administration of higher doses of drug. As a result, localizing drug activity would improve the efficacy of the treatment, reduce off-target effects, enhance the patients' quality of life and therapeutic compliance. Light is a powerful tool in this respect. ${ }^{9,10}$ It offers unparalleled opportunities as a non-invasive regulatory signal for biological and pharmacological applications because it can be delivered with high precision regarding space, time, intensity and wavelength, it generally interacts weakly with tissue (except for scattering), and causes negligible toxicity for the human body. ${ }^{11,12,13,14,15}$ Several cancer treatments relying on the use of light have emerged during the last decades. ${ }^{8}$ One of those, known as photodynamic therapy (PDT), is already well established and clinically applied. The procedure involves administration of a photosensitizer that forms reactive oxygen species (ROS) upon irradiation with light at a specific wavelength, leading to direct tumor cell death, damage to the microvasculature and induction of an inflammatory reaction. ${ }^{16}$ Although PDT is on the rise as a cancer treatment modality, its dependence on the presence of molecular oxygen ${ }^{17,18,19}$ and the 
frequent occurrence of unbearable inflammation-induced pain represent important drawbacks that can remarkably limit its applicability and success rate. ${ }^{20,21}$ Actually, oxygenindependent treatments relying on the use of photoactivatable metal complexes ${ }^{22}$ or photoactivatable prodrugs ("caged compounds" $)^{23,24,25}$ have emerged as an alternative, but in these cases the irreversibility of activation of the cytotoxic species and their subsequent diffusion outside the intended site of action represent in turn major disadvantages. a<smiles>CCOCCN(C)c1ccc(C(=O)N[C@@H](CCC(=O)O)C(=O)O)cc1</smiles>

C

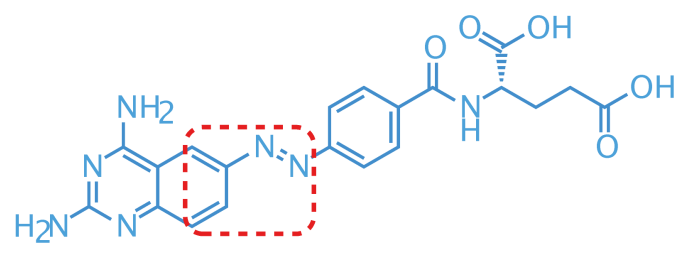

trans-Phototrexate b

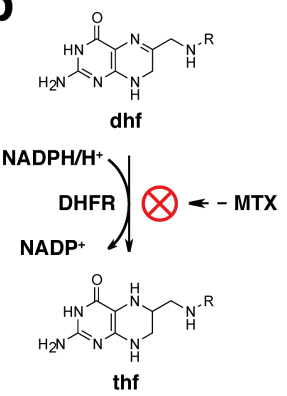

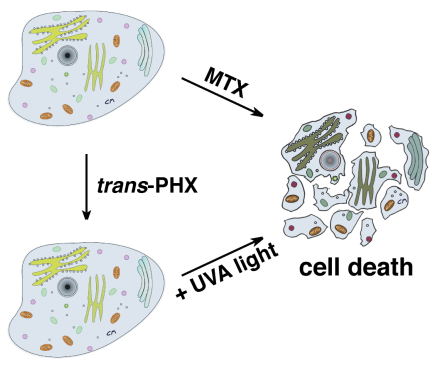

$\underset{\text { blue light }}{\stackrel{\text { UVA light }}{\rightleftharpoons}}$<smiles>Nc1nc(N)c2cc(/N=N\c3ccc(C(=O)N[C@@H](CCC(=O)O)C(=O)O)cc3)ccc2n1</smiles>

d

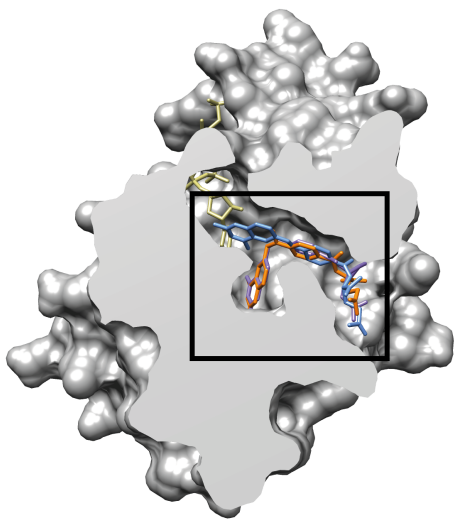

e

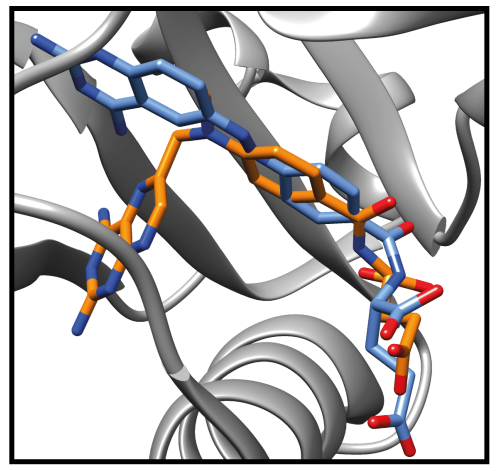

f

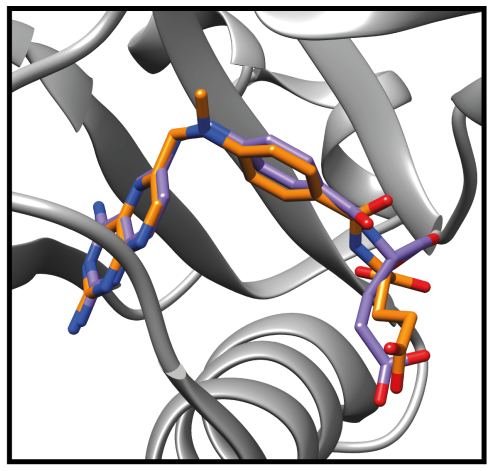

Figure 1. Mechanism of action of methotrexate (MTX) and design strategy of phototrexate (PHX). (a) Chemical structure of the DHFR inhibitor MTX. (b) Mechanism of action of DHFR inhibitors (left) and cartoon representation of cell death (right) induced by a conventional versus a light-regulated DHFR inhibitor: the latter should be constitutively inactive and allow DHFR inhibition upon illumination at the proper wavelength. (c) Chemical structures of phototrexate in the trans (in the dark and under blue/white light illumination, structure in blue) and cis (under UVA illumination, structure in purple) configurations. The red dotted rectangle defines the moiety involved in the azologization strategy. (d) Crystal structure of the human DHFR in complex with MTX (in orange) and the cofactor NADPH (in khaki) (PDB 1U72, protein surface representation with sectioning to reveal the active site pocket). The folded cis-like configuration adopted by MTX in the active site is shown. Docked structures of trans (in blue) and cis (in purple) phototrexate are also represented. (e),(f) Zoomed views of trans (in blue) and cis (in purple) phototrexate (best docked poses) superimposed on the crystallographic structure of MTX (in orange) in the active site of the human DHFR. In our docking simulations (see SI for details), unlike its trans counterpart, cisphototrexate shows a high degree of overlap to the crystallographic pose of MTX. Nitrogen and oxygen atoms of the ligands are colored in blue and red, respectively. 
Overall, current strategies to improve the safety and efficacy of cancer treatment are promising, but the availability of truly tumor-selective therapies remains an unmet medical need. Photopharmacology, which relies on the use of reversibly photoswitching drugs, is well-suited to this task. ${ }^{26,27,28,29}$ Several light-regulated compounds have been described to reversibly control a diversity of proteins. They are obtained by introduction of a molecular photoswitch into the structure of an existing drug in order to alter its pharmacological properties upon illumination. ${ }^{27}$ Azobenzenes, and more in general arylazo compounds, have been widely employed as molecular photoswitches because of their favorable characteristics, namely design flexibility, ease of synthesis, large changes in geometry upon isomerization, relatively stable photostationary states and high quantum yields, fast photoisomerization rates, spontaneous relaxation to the thermodynamically more stable configuration (T-type photoswitch), and low photobleaching rates. ${ }^{15}$ Furthermore, the use of compounds bearing arylazo moieties has been proven safe in humans, including approved drugs and food colorants. ${ }^{27}$ Examples of bioactive molecules with photoswitchable efficacy include $G$ protein-coupled receptor modulators, ${ }^{30,31,32}$ ion channel modulators, ${ }^{33}$ enzyme inhibitors, ${ }^{34}$ and inhibitors of protein-protein interactions. ${ }^{35}$ Recently, a number of photoswitchable cytotoxic agents acting on different biological targets have also been reported, thus demonstrating an increasing attention to this approach. ${ }^{9,36,37}$ Indeed, photopharmacology has a transformative potential, by bringing together the advantages of targeted therapies and light-based oxygen-independent therapies in order to improve the safety and efficacy of anticancer treatments. Of relevance are the results obtained with photoswitchable combretastatin A-4 analogues, named photostatins or azo-combrestatins, acting as tubulin polymerization inhibitors. Four research groups have independently published their findings on the efficacy of such derivatives as photoswitchable light-regulated cytotoxic agents in different cell lines. ${ }^{38,39,40,41}$ Another example was published by Szymanski and coll. ${ }^{10}$ Their photoswitchable histone deacetylase inhibitor displayed a remarkable cytotoxicity in vitro and emerges as a putative lead compound for photopharmacological chemotherapy.

Here, we have taken one step further towards the development of light-regulated cancer chemotherapy by photocontrolling the in vitro antiproliferative action and in vivo toxicity of a target-specific, widely prescribed chemotherapeutic agent. For that purpose, we first performed a selection of protein targets and anticancer drugs complying with several criteria: (1) clinically validated target, (2) wide application in high-incidence diseases, (3) in use to treat cancer forms affecting organs or tissues potentially accessible to light, either directly or through natural orifices (e.g., skin, bladder, intestine), (4) a drug safety profile including severe systemic side effects which currently limit its use, (5) drug structure suitable for photoregulation with minimal modifications, (6) biological inertness in the dark-relaxed form, (7) compatibility with phototherapy and/or used as adjunctive treatment, and (8) synthetic accessibility. We extensively documented the candidates using medical and scientific literature $e^{42,43}$ and public databases, including DrugBank, ${ }^{44}$ ChEMBL, ${ }^{45}$ Protein Data Bank, ${ }^{46}$ and Drugs.com. ${ }^{47}$ This survey ranked methotrexate (MTX) as one of the most prominent drugs matching the identikit to develop a lightregulated cytotoxic agent (Figure 1a).
MTX belongs to a specific class of chemotherapeutic agents known as antimetabolites, which kill hyperproliferative cells by targeting their metabolism. Although antimetabolites have been known for long, they are still actively developed for cancer chemotherapy and are widely used in the treatment of diverse human tumors. ${ }^{48,49}$ Notably, MTX inhibits dihydrofolate reductase (DHFR), which catalyzes the conversion of dihydrofolate (dhf) to tetrahydrofolate (thf), a key step in folate metabolism (Figure 1b). Interrupting folate metabolism compromises thymidine synthesis, and interferes with purine synthesis and other reactions involving single-carbon transfers, leading to cell death (Figure 1b) ${ }^{48,50}$ MTX is commonly used alone or in combination with other antineoplastic drugs in the treatment of certain cancers including but not limited to breast cancer, epidermoid cancers of the head and neck, lung cancer (particularly squamous cell and small cell types) and bladder cancer. In addition, because of its immunosuppressive properties, it is also indicated in the symptomatic control of severe, recalcitrant, disabling psoriasis, and in the management of severe, active rheumatoid arthritis. ${ }^{51}$ However, the importance and ubiquity of the MTX target (DHFR) in all cell types is at the origin of a poor drug safety profile that often leads to unbearable side effects requiring dose reduction or treatment cessation. The most frequently reported adverse reactions include ulcerative stomatitis, leukopenia, nausea, abdominal distress, malaise, undue fatigue, chills and fever, dizziness, and decreased resistance to infection. ${ }^{52}$

Besides the motivation to enhance the pharmaco-toxicological profile of MTX, two reasons supported the feasibility of photocontrolling MTX activity. The first one is that azobenzene analogs of the MTX structure can be readily designed. There are two general strategies to devise arylazo-based lightregulated drugs: (1) by elongation of the drug structure with an arylazo moiety ("azo-extension"), ${ }^{53,54,55}$ and (2) by incorporation of an arylazo moiety into the pharmacophore of the drug ("azologization"). ${ }^{30,56}$ MTX is suitable for the latter, which is generally preferred because it requires minimal modifications of the original structure, thus maintaining the druglikeness of the parent compound, and largely preserving its pharmacokinetic and pharmacodynamic properties (Figure $1 \mathrm{c}$ ). ${ }^{57}$ The second reason is that the activity of MTX azologs is expected to be higher in their cis state ("cis-on") than in the most thermodynamically stable trans configuration. This prediction is based on the crystal structure of the ternary complex of MTX with the wild-type human DHFR and the cofactor NADPH (PDB 1U72), ${ }^{58}$ in which MTX is bound to the enzyme active site in a bent "cis-like" configuration (Figure 1d-f). When designing bioactive azologs, a "trans-off/cis-on" behavior is often desired because such compounds can be safely applied (e.g., systemically) in the inactive relaxed form and they can be photoactivated at the required times and locations. Thus, MTX azologs fulfill all the above-mentioned structural and (photo)pharmacological design requirements of a cis-on druglike antifolate candidate for light-regulated chemotherapy.

\section{RESULTS AND DISCUSSION}

Design strategy and synthesis. MTX structural azologs could be designed by replacing the methyleneamino bridge between the diaminopteridine and the benzamido moieties with an azo $(-\mathrm{N}=\mathrm{N}-)$ group. However, before applying this 
strategy, we reviewed the structure-activity relationships of MTX and related analogs in order to define the role of this bridge. We found out that a great variety of structural variants of the bridge are tolerated, and some of them can even favor the accumulation and persistence of the drug in responsive tumor cells rather than in normal proliferative tissues. ${ }^{59,60}$ Moreover, it is reported that 5,8-dideaza (quinazoline) DHFR inhibitors obtained by isosteric replacement of the nitrogen atoms at positions $\mathrm{N} 5$ and $\mathrm{N} 8(-\mathrm{N}=\mathrm{C})$ of the pteridine ring with $-\mathrm{CH}=$ groups are endowed with comparable inhibitory activity. ${ }^{61,62}$ In this regard, generating a diazo bond at position C6 of a quinazoline is expected to be more synthetically accessible than at a pteridine, while still providing a strong DHFR inhibitor. Thus, we conceived a putative photochromic DHFR inhibitor by MTX azologization and two bioisosteric substitutions, and named it phototrexate (PHX, Figure 1c).

In order to achieve the desired biological properties (Figure 1b), phototrexate should be constitutively inactive in its most thermodynamically stable configuration (trans isomer, darkadapted state), and it should mimic the pharmacological effects of the parent drug (MTX) upon illumination at the proper wavelength ( $c i s$ isomer). To test the feasibility of our design, we performed molecular docking simulations of cis- and trans-phototrexate in the atomic structure of the human DHFR (PDB 1U72). ${ }^{58}$ The results (see also SI for details) show that cis-phototrexate binds to the active site of the target enzyme in a mode that strictly mimics the orientation and conformation adopted by MTX in its crystallographic pose, with a root mean square deviation (RMSD) of $0.5 \AA$ between the best-scoring pose of cis-phototrexate and the crystallographic pose of MTX, and a predicted binding energy of $-10.8 \mathrm{kcal} / \mathrm{mol}$ (Figure 1f). In contrast, trans-phototrexate displayed a set of binding poses that barely overlapped with MTX, with a RMSD of $4.8 \AA$ between its best docked pose and the crystallographic pose of MTX, and a predicted binding energy of $-9.3 \mathrm{kcal} / \mathrm{mol}$ (Figure 1d-f). Overall, these computational results lend support to the assumption of a "cis-on" antifolate behavior of phototrexate and encouraged pursuing the synthesis of the target compound.

Phototrexate was prepared via a convergent 4-step synthetic route starting from commercially available compounds (Scheme 1). To generate the diazo bond of phototrexate, we planned to perform a coupling reaction between its quinazoline and benzamido building blocks. The required amine and nitroso intermediates were therefore prepared for this purpose. A catalytic hydrogenation of 6-nitroquinazoline-2,4-diamine afforded the desired quinazoline-2,4,6-triamine (1) in high yield $\quad(98 \%), \quad$ while $(S)$-diethyl 2-(4nitrosobenzamido)pentanedioate (2) was obtained by oxidation of the corresponding amine with Oxone ${ }^{\circledR}$ in a biphasic system of dichloromethane and water. These intermediates were then combined under Mills conditions (55\% yield) to give $\quad(S, E)$-diethyl 2-(4-((2,4-diaminoquinazolin-6yl)diazenyl)benzamido)pentanedioate (3), which was finally hydrolyzed in alkaline conditions to provide phototrexate in high yield (95\%). Physicochemical characterization of each intermediates and the final compound is provided in the SI file.<smiles>Nc1nc(N)c2cc([N+](=O)[O-])ccc2n1</smiles><smiles>CCOC(=O)CC[C@H](NC(=O)c1ccc(N)cc1)C(=O)OCC</smiles>

$50 \mathrm{psi} \mathrm{H}_{2}$
$10 \% \mathrm{Pd}^{-C}$
$\mathrm{AcOH}, \mathrm{DMF}$
$5 \mathrm{~h},(98 \%)$<smiles>Nc1ccc2nc(N)nc(N)c2c1</smiles>

1

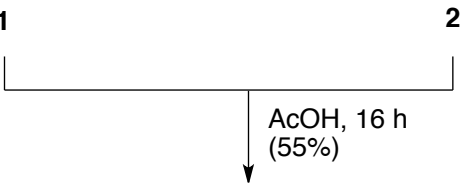<smiles>CCOC(=O)CC[C@H](NC(=O)c1ccc(N=Nc2ccc3nc(N)nc(N)c3c2)cc1)C(=O)OCC</smiles>

3

$$
\mid \begin{aligned}
& 1 \mathrm{M} \mathrm{NaOH}, \mathrm{EtOH} \\
& 0^{\circ} \mathrm{C} \rightarrow \mathrm{rt}, 1 \mathrm{~h} \\
& (95 \%)
\end{aligned}
$$

\section{Phototrexate}

Scheme 1. The chemical synthesis of phototrexate.

Photochemical characterization. An essential requirement for using phototrexate as a light-dependent DHFR inhibitor is that it efficiently responds to light, which means that it can be quickly photo-isomerized between two different configurations with a relatively high degree of photoconversion (trans/cis ratio). UV/Vis absorption studies showed a robust photochromic behavior in aqueous solution. Phototrexate can be effectively isomerized from trans to cis with UVA light (375 nm), and back-isomerized from cis to trans with blue $(460 \mathrm{~nm})$ or white light (Figure S2). This transition is reversible and can be repeated several times without any noticeable loss of absorption (Figure S3). We then quantified by ${ }^{1} \mathrm{H}$ NMR analysis the ratio between the two isomers at the photostationary state (PSS). The amount of the thermodynamically less stable cis isomer shifted from $0 \%$ (dark-adapted state) to $76 \%$ upon illumination with $375 \mathrm{~nm}$ light for 30 minutes (4.6 $\mathrm{J} / \mathrm{cm}^{2}$, Figure $2 \mathrm{a}$ ). In addition, as phototrexate is a T-type photoswitch, we also studied its spontaneous thermal relaxation (from cis to trans) in dark conditions. The measured half-life was approximately $690 \mathrm{~min}$ at $25^{\circ} \mathrm{C}$ and $236 \mathrm{~min}$ at $37{ }^{\circ} \mathrm{C}$ in aqueous solution (Figure $2 \mathrm{~b}$ ). Finally, we investigated the time dependence (Figure 2c) and the light fluence dependence (Figure 2d) of the photoisomerization (from trans to cis) under UVA $(375 \mathrm{~nm})$ illumination. These results show that maximal photoconversion is achieved with a fluence greater than 0.6 $\mathrm{J} / \mathrm{cm}^{2}$. Overall, the photochromic properties of phototrexate 
show that it can be efficiently and reversibly photoisomerized using UVA and white light, and that it has a stability of several hours in the dark at the normal human body temperature.

a

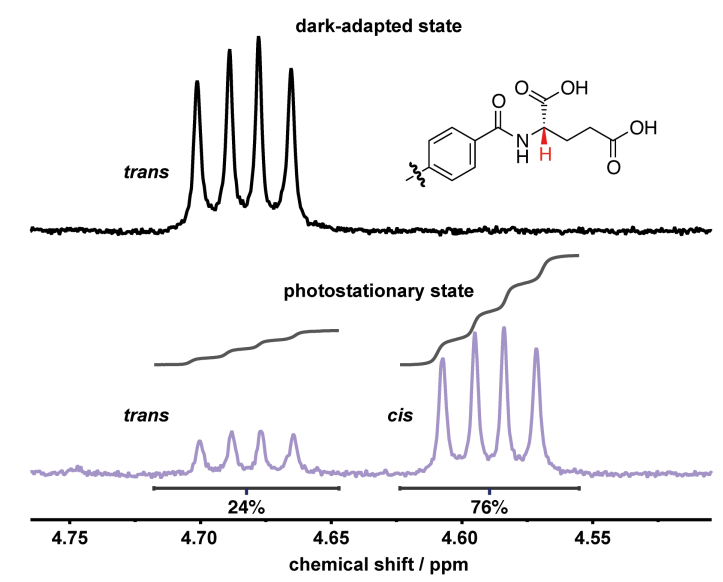

C

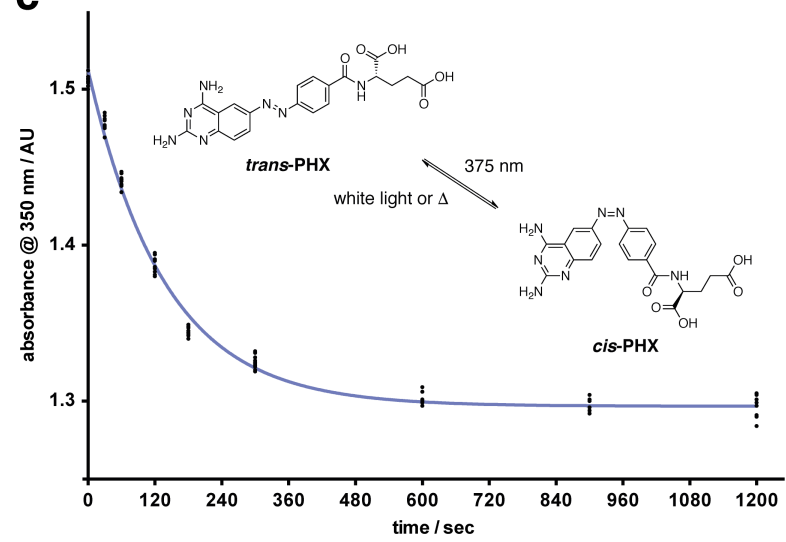

b

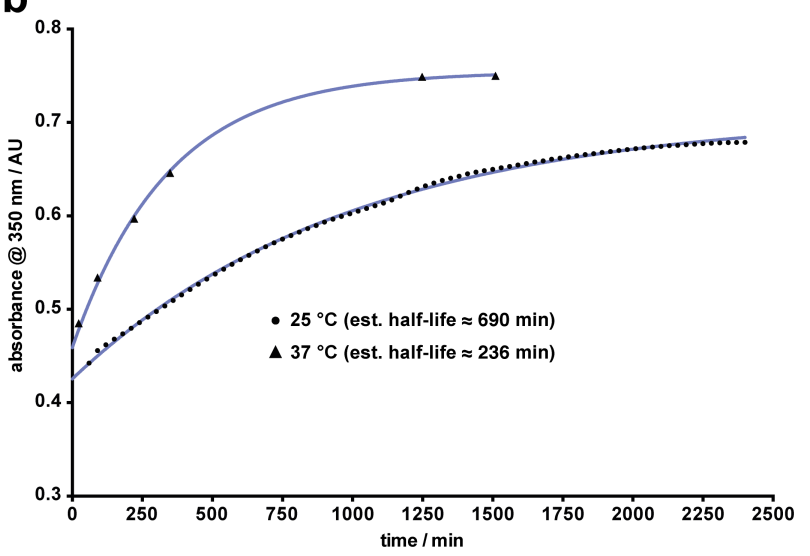

d

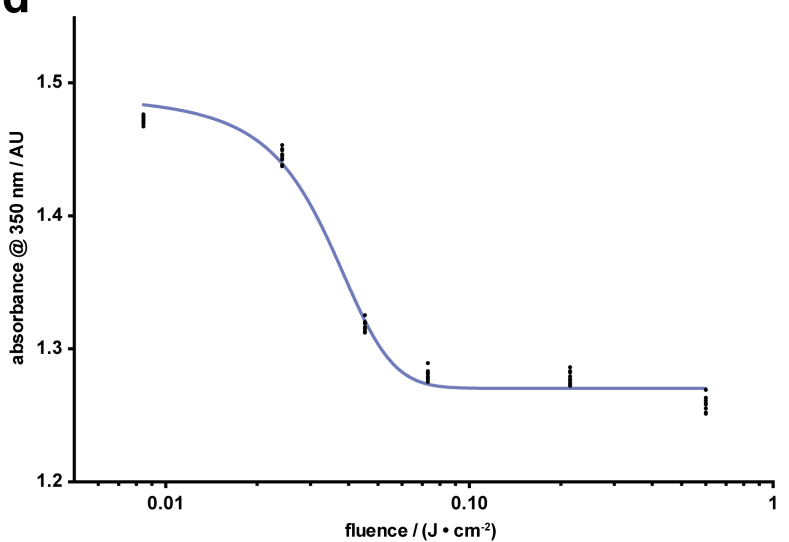

Figure 2. Photochemical characterization of phototrexate (PHX). (a) Composition of the photostationary state achieved after illumination with $375 \mathrm{~nm}$ light for $30 \mathrm{~min}\left(4.61 \mathrm{~J} / \mathrm{cm}^{2}\right)$ determined by ${ }^{1} \mathrm{H}$ NMR (5 mM in methanol-d4). (b) Thermal relaxation of phototrexate (from trans to cis) in the dark at $25^{\circ} \mathrm{C}(50 \mu \mathrm{M})$ and $37^{\circ} \mathrm{C}(100 \mu \mathrm{M})$, measured by absorbance at 350 nm. Half-lives were estimated using the one-phase decay function in GraphPad Prism 6 (nonlinear regression curve fitting). (c) Time dependence of photoisomerization of phototrexate (from trans to cis) under $375 \mathrm{~nm}$ light measured by absorbance of a $200 \mu \mathrm{M}$ solution at $350 \mathrm{~nm}$. Data were fitted to the one-phase decay function in GraphPad Prism 6 (nonlinear regression curve fitting). (d) Light fluence dependence of photoisomerization of phototrexate (from trans to cis) under $375 \mathrm{~nm}$ light measured by absorbance of a $200 \mu \mathrm{M}$ solution at $350 \mathrm{~nm}$. Data were fitted to the four-parameter dose-response curve in GraphPad Prism 6 (nonlinear regression curve fitting).

In vitro photopharmacological characterization. After obtaining the target chemical compound with the desired photochromic properties, we characterized its biological activity using in vitro assays. For convenience, in the text we will refer to the all-trans form of phototrexate in the dark-adapted state as trans-phototrexate, and to the cis-enriched form (obtained at the PSS upon illumination at $375 \mathrm{~nm}$ ) as cis-phototrexate. We first assessed the ability of the two photoisomers of phototrexate to inhibit the purified target enzyme. To this end, we quantified DHFR activity using a colorimetric assay (see SI for details) and observed significant differences between the two isomers at $10 \mathrm{nM}$ and $100 \mathrm{nM}$, with $10 \mathrm{nM}$ cisphototrexate inhibiting DHFR activity more strongly (70\%) than the trans isomer at the same concentration (21\%) (Figure 3a). The stronger inhibition capacity exhibited by cisphototrexate thus matched with our molecular docking simulations and fulfilled the design requirements of a cis-on antifolate (Figure 1).
We next examined the cytotoxicity of phototrexate in cells using a MTT [3-(4,5-dimethylthiazol-2-yl)-2,5diphenyltetrazolium bromide] assay for cell viability. HeLa cervical cancer cells were incubated with trans- and cisphototrexate for 24 hours at $37^{\circ} \mathrm{C}$, and cell viability was subsequently measured. Methotrexate was tested in the same conditions as the positive control. Cells were also incubated in vehicle solution (negative control wells) and used for normalization of cell viability. The cis isomer caused significantly lower viability to HeLa cells than the trans isomer (Figure 3b), in line with the results obtained in the enzyme inhibition assay. Notably, $1 \mu \mathrm{M}$ cis-phototrexate reduced viability to $27 \%$, whereas the trans isomer barely altered it (88\%). At the maximum concentration tested $(100 \mu \mathrm{M})$, only $23 \%$ of cells survived to cis-phototrexate, against $78 \%$ for trans-phototrexate. A remarkable difference in $\mathrm{IC}_{50}$ was estimated for the two isomers, with a value of about $6 \mathrm{nM}$ for the cis and $34 \mu \mathrm{M}$ for the trans compound. Interestingly, cis-phototrexate was as ef- 
fective as the reference drug methotrexate, which reduced cell viability to $40 \%$ at $1 \mu \mathrm{M}$ and $33 \%$ at $100 \mu \mathrm{M}$. Overall, these results indicate that MTX and cis-phototrexate have a similar cytotoxicity profile in HeLa cancer cells, whereas transphototrexate is nearly inactive at the same concentrations. Considering that cis-phototrexate has a relatively short halflife of thermal relaxation in the test conditions $(\sim 236$ minutes at $37^{\circ} \mathrm{C}$, for an incubation time of 24 hours), the difference observed between cis and trans in terms of cytotoxicity is remarkable. The effect of cis-phototrexate can be realistically ascribed to the disruption of the folate pathway, since this process plays an essential role in the synthesis of DNA and RNA, in the metabolism of amino acids, and ultimately in the process of cell division. Nevertheless, a diversity of effects of the two photoisomers on other biochemical pathways beside the folate pathway (e.g., inhibition of thymidylate synthase and glycinamide ribonucleotide transformylase), ${ }^{63}$ dissimilarities in cellular uptake and polyglutamation, ${ }^{63}$ or differences in offtarget binding to intracellular structures, ${ }^{64}$ as reported for MTX, should not be ruled out and could account for the differences in drug sensitivity between assays with purified target protein and assays on more complex systems, such as cells and living organisms. a

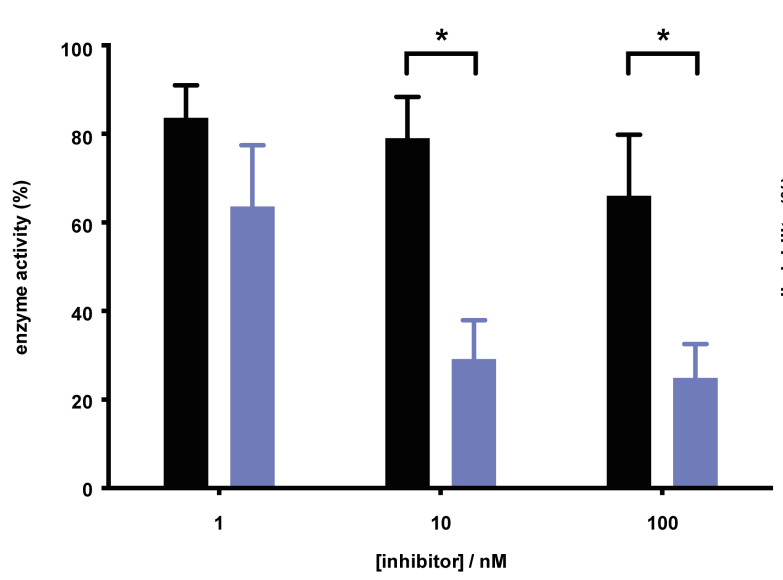

b

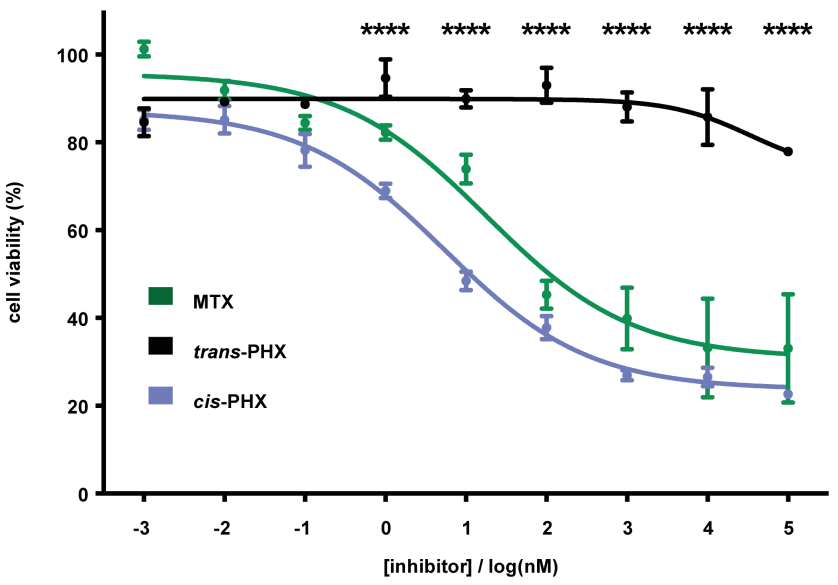

Figure 3. In vitro pharmacological characterization of phototrexate (PHX). (a) Inhibition of DHFR activity at $1 \mathrm{nM}, 10 \mathrm{nM}$ and 100 $\mathrm{nM}$ of trans (black) and cis (violet) phototrexate. Data are means of at least three independent experiments in triplicate or quadruplicate \pm SEM. Results were analyzed by two-way ANOVA with Bonferroni's multiple comparisons test for statistical significance (GraphPad Prism 6 ; adjusted p-value $(*) \leq 0.05$ ). (b) HeLa cell viability assay at different concentrations of MTX, cis-phototrexate and trans-phototrexate. The relatively low vertical span and Hill coefficient of the sigmoidal curves of MTX and cis-phototrexate might be ascribed to short exposure time of the cells to the drugs, ${ }^{65}$ saturation of the carrier-mediated uptake, and partitioning of the inhibitors into an inaccessible form at the higher concentrations. ${ }^{66}$ Data are means of at least three independent experiments in triplicate or quadruplicate \pm SEM. Results were analyzed by two-way ANOVA with Bonferroni's multiple comparisons test for statistical significance (GraphPad Prism 6; adjusted $p$-value $(* * * *) \leq 0.0001$ for trans-phototrexate versus cis-phototrexate).

Disruption of folate metabolism in zebrafish larvae. In view of the efficacy and light-regulation of phototrexate antiproliferative properties in vitro, we evaluated the performance of our compound in a small animal model. Since DHFR inhibitors disrupt folate metabolism, they have a high impact at early stages of animal development. Zebrafish (Danio rerio) embryos have been used to characterize the effects of methotrexate ${ }^{67}$ and thus constitute an excellent model system to investigate the effects of trans- and cis-phototrexate in vivo. Zebrafish fertilized eggs were incubated within 5 hours post fertilization (hpf) in UV purified water containing trans- or cis-phototrexate $(200 \mu \mathrm{M})$ for 5 days at $28{ }^{\circ} \mathrm{C}$. MTX was also tested as the positive control $(200 \mu \mathrm{M})$. Each embryo (normally until 48-72 hpf) and hatched larva (from 48-72 hpf) was ob- served daily to monitor changes in their anatomy and behavior. The four groups (vehicle, MTX, cis-phototrexate, transphototrexate) were compared at 72 and $96 \mathrm{hpf}$, when certain anatomical and behavioral traits become easier to observe (Figure 4a-d). In particular, healthy hatched larvae reach the stage of protruding-mouth at $72 \mathrm{hpf},{ }^{68}$ with a length of about $3.5 \mathrm{~mm}$. Normal anatomical traits include iridiophores in the yolk and half of the eye, with yellow pigmentation on the head and in the dorsal part and black pigmented lateral and ventral stripes from the head to the tail, as well as observable heart beat and blood flow (see SI for details). ${ }^{68}$ 

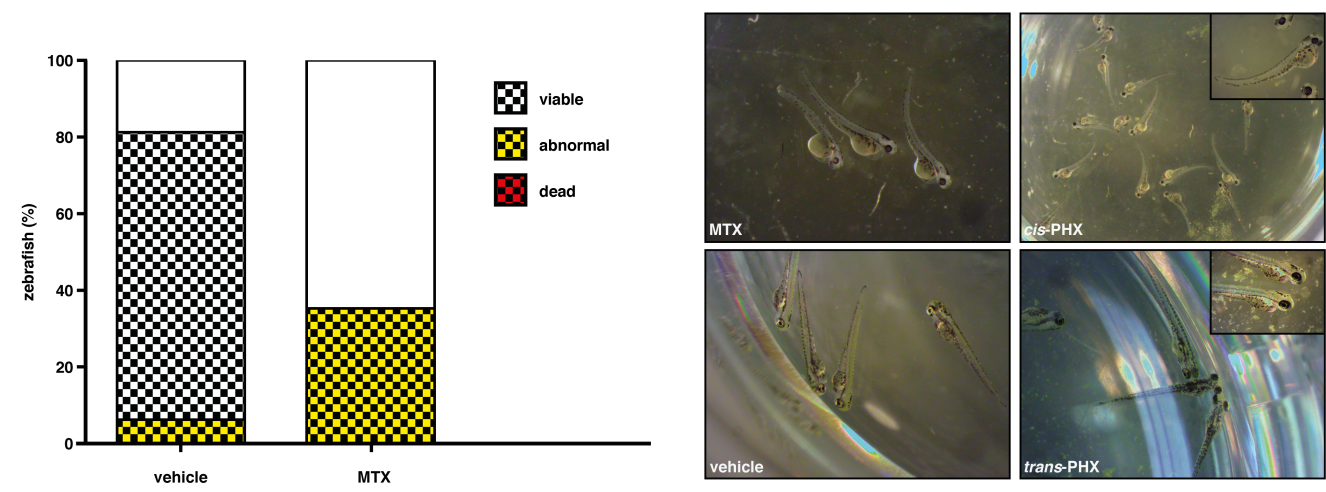

C

72 hpf

d

$96 \mathrm{hpf}$
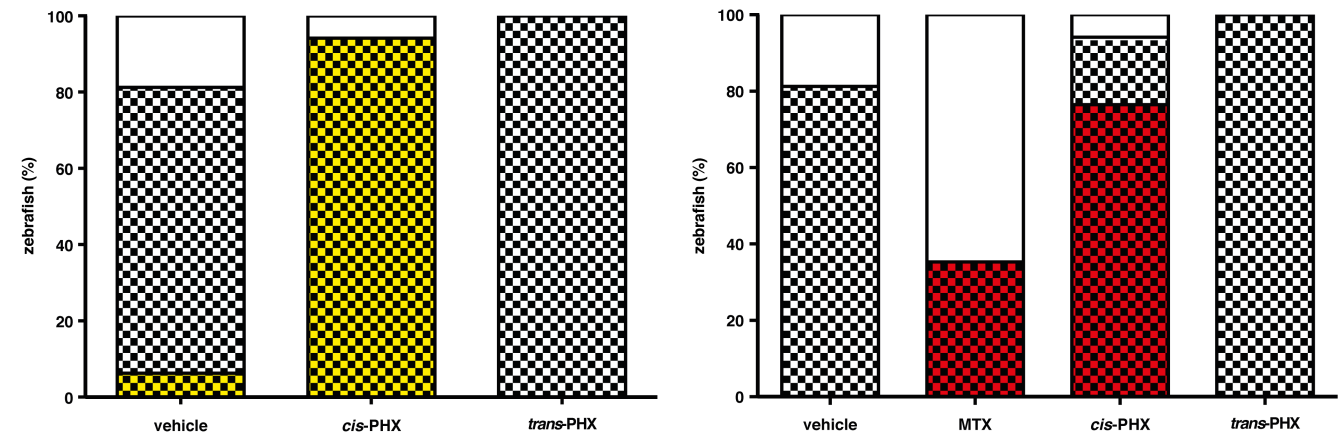

e

$96 \mathrm{hpf}$

f

$96 \mathrm{hpf}$
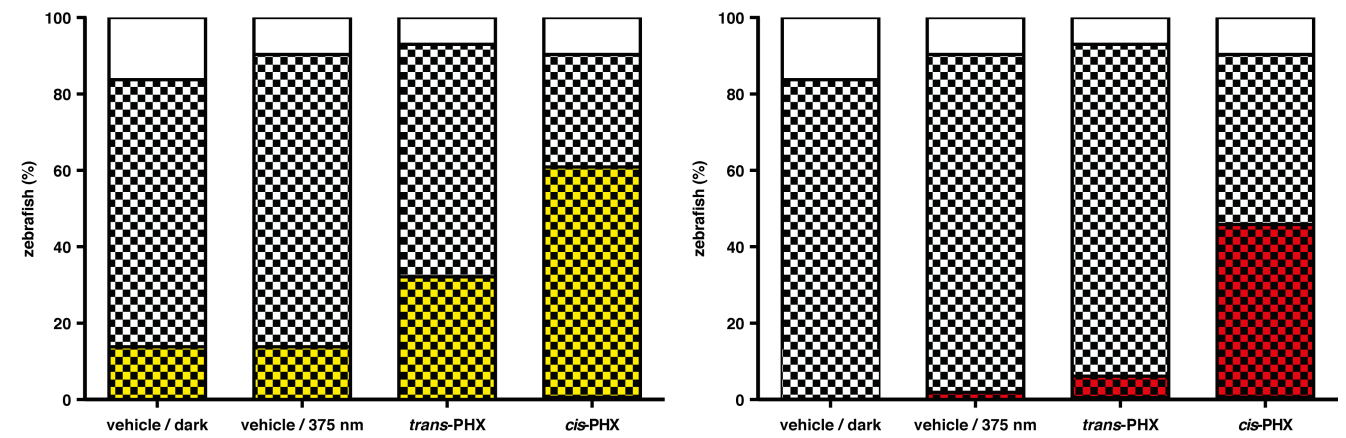

Figure 4. Effects of phototrexate (PHX) on zebrafish development, viability and mortality. Experiment 1, panels a-d: cis-phototrexate was obtained by external pre-illumination with $375 \mathrm{~nm}$ light (see SI for details). (a), (c) Anatomical profiles of zebrafish at $72 \mathrm{hpf}$ after treatment with vehicle (DMSO $2 \%)$, MTX $(200 \mu \mathrm{M})$, cis-phototrexate $(200 \mu \mathrm{M})$, and trans-phototrexate $(200 \mu \mathrm{M})$. The graph shows the percentage of zebrafish treated versus the treatment group, superimposing the number of abnormal larvae (black and yellow checkered sections) to the number of viable embryos (checkered sections) and to the total number of fertilized embryos (whole columns). (b) Illustrative pictures of individual larvae from each treatment group at $72 \mathrm{hpf}$, showing the observable aberrant developmental traits in the MTX and cis-phototrexate groups. (d) Mortality of zebrafish at $96 \mathrm{hpf}$ after treatment with vehicle (DMSO 2\%), MTX (200 $\mu$ M), cis-phototrexate $(200 \mu \mathrm{M})$, and trans-phototrexate $(200 \mu \mathrm{M})$. The graph shows the percentage of zebrafish treated versus the treatment group, superimposing the number of dead zebrafish (black and red checkered sections) to the number of viable embryos (checkered sections) and to the total number of fertilized embryos (whole columns). Experiment 2, panels e-f: cis-phototrexate was obtained by in situ illumination with 375 $\mathrm{nm}$ light in the presence of zebrafish. (e) Anatomical profiles of zebrafish at $96 \mathrm{hpf}$ after treatment with vehicle/dark (DMSO $2 \%$, no illumination), vehicle $/ 375 \mathrm{~nm}$ light (DMSO $2 \%, 0.61 \mathrm{~J} / \mathrm{cm}^{2}, 4 \mathrm{~min}$ illumination twice a day), trans-phototrexate (200 $\mu \mathrm{M}$, dark), and cisphototrexate $\left(200 \mu \mathrm{M}, 0.61 \mathrm{~J} / \mathrm{cm}^{2}, 4 \mathrm{~min}\right.$ illumination twice a day). The graph shows the percentage of zebrafish treated versus the treatment group, superimposing the number of abnormal larvae (black and yellow checkered sections) to the number of viable embryos (checkered sections) and to the total number of fertilized embryos (whole columns). (f) Mortality of zebrafish at 96 hpf after treatment with vehi-

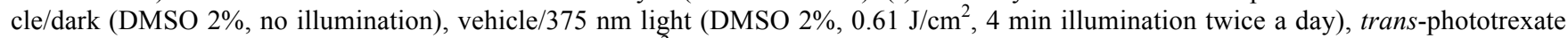
$\left(200 \mu \mathrm{M}\right.$, dark), and cis-phototrexate $\left(200 \mu \mathrm{M}, 0.61 \mathrm{~J} / \mathrm{cm}^{2}, 4 \mathrm{~min}\right.$ illumination twice a day). The graph shows the percentage of zebrafish treated versus the treatment group, superimposing the number of dead zebrafish (black and red checkered sections) to the number of viable embryos (checkered sections) and to the total number of fertilized embryos (whole columns). 
Embryos treated with MTX presented a low viability at 24 hpf, with six out of seventeen total embryos viable (35\%, Figure 4a). At $72 \mathrm{hpf}$, all six individuals presented three abnormalities: a deficient iridiophore ocular pigmentation, with complete black colored eyes and a deficient black body stripe patterns; an abnormal volume of the cardiac cavity; and tail angle deviations, with prominent bent or curved tails (Figure $4 b)$. Viability in the vehicle and cis-phototrexate groups remained within normal batch levels (only two and one nonviable embryos, respectively), and perfect in the transphototrexate group (Figure $4 \mathrm{~b}-\mathrm{c}$ ). In the vehicle group, only one larva presented malformation, a non-hatched individual with unformed tail. Cis-phototrexate produced a toxicity profile similar to that of the reference compound MTX, generating comparable developmental abnormalities at $72 \mathrm{hpf}$ together with a high rate of mortality at 96 hpf (Figure 4d). In stark contrast, trans-phototrexate showed a lower general toxicity, as it did not produce any observable abnormality at $72 \mathrm{hpf}$ and no mortality at $96 \mathrm{hpf}$.

Next, we tested the performance of phototrexate by photoswitching its pharmacological effects in situ. We used again zebrafish as animal model. Four groups of zebrafish fertilized eggs ( $n=48$ in each group) were incubated within 5 hours post fertilization (hpf) in UV purified water containing the drug vehicle (DMSO $2 \%$, groups 1 and 2) or phototrexate (200 $\mu \mathrm{M}$, groups 3 and 4 ) for 5 days at $28^{\circ} \mathrm{C}$ (Figure 4e-f). Groups 1 and 3 (vehicle and trans-phototrexate) were constantly kept in the dark, whereas group 4 was illuminated twice a day with $375 \mathrm{~nm}$ light $\left(0.61 \mathrm{~J} / \mathrm{cm}^{2}, 4 \mathrm{~min}\right.$ illumination) to generate a significant amount of cis-phototrexate directly in the fish medium. Group 2 (vehicle) underwent a parallel and identical protocol of illumination to determine whether the direct exposure to UVA light could intrinsically affect fish development and survival. The viability of individual embryos and hatched larvae was monitored daily. The four groups were finally examined at $96 \mathrm{hpf}$ to assess the abnormality and mortality profiles (Figure 4e-f). All groups presented normal development and a good viability at $24 \mathrm{hpf}$ (greater than $80 \%$ ). When comparing the number of larvae with anatomical malformations and abnormalities to the total number of embryos, both vehicle groups (dark and UVA) presented $12 \%$ of abnormal individuals (with anomalous volume of the cardiac cavity and sidewise position) at $96 \mathrm{hpf}$. In the trans-phototrexate group, 30\% of the larvae were affected by abnormalities, including cardiac cavity and curved tails, while $60 \%$ of the individuals were found with a similar profile of abnormalities and lower pigmentation in the group where phototrexate was switched on (cis isomer) with UVA light (Figure 4e). Analyzing the mortality profile at $96 \mathrm{hpf}$, the vehicle/dark group did not show any loss of individuals, and only $2 \%$ were found dead in the vehicle/UVA group. Therefore, the light intensity, light wavelength and illumination protocol adopted for the experiment were well tolerated by the fish. Importantly, we found out that only $6 \%$ of the total embryos (7\% of the viable ones) were dead in the trans-phototrexate group against the $46 \%$ of the total embryos (51\% of the viable ones) in the cis-phototrexate group (Figure $4 \mathrm{f}$ ). Thus, the abnormality and mortality rate in the fish wells in which phototrexate was switched on with light was remarkably higher than those maintained in the dark (two- and eight-fold, respectively). These results (Figure 4e-f) are in good agreement with those obtained in the previous experiment (Figure 4a-d) and demonstrate that the cytotoxic ef- fects of phototrexate can be turned on also by direct illumination of living animals treated with its trans "inactive" form.

Overall, our experiments in vivo, together with the in vitro assays (Figure 3), indicate that photoactivated cis-phototrexate has a pharmacological profile of a potent antifolate drug, whereas it is almost inert in its trans configuration. Slight discrepancies observed between MTX and cis-phototrexate activities on cellular and organism assays might be related in part to non-identical off-target activities.

\section{CONCLUSIONS}

In summary, we have rationally designed, synthesized, and characterized phototrexate, the first light-regulated inhibitor of the human DHFR, and we have demonstrated its antifolate and antiproliferative properties using in vitro and in vivo assays. Phototrexate has been derived by azologization and bioisosteric substitutions of methotrexate, a DHFR inhibitor that is extensively used to treat many forms of cancer, severe psoriasis and rheumatoid arthritis. In this way, the activity, druglikeness and other determinants of the pharmacokinetic profile of the parent compound should be retained. Our results show that phototrexate can be switched on with UVA light to become a potent antifolate drug comparable to methotrexate, and it can be switched off with blue or white light and by thermal relaxation in the dark. This profile offers the opportunity of inhibiting cellular proliferation in a target-specific manner and at confined locations, while reducing these effects in other regions. In addition, light-activated phototrexate self-deactivates (thermally relaxes) upon diffusing away from the treated region. Thus, phototrexate constitutes a proof-of-concept to develop photoswitchable antimetabolite drugs, and makes it possible to explore photopharmacological chemotherapies with reduced systemic side effects and with enhanced tolerability and antineoplastic efficacy. Major advantages of such lightbased approach over the existing ones are that the effects of photoswitchable chemotherapeutics like phototrexate are target specific (cf. PDT), oxygen-independent (cf. PDT), and reversible (cf. PDT, photoactivatable metal complexes, and photocleavable prodrugs). Small molecules like phototrexate could be administered topically in their active form, or systemically in their inactive form followed by precise photoactivation only at the lesion area. Target regions might be tissues that can be exposed to illumination, like the skin and the digestive, respiratory and reproductive tracts. Notably, the envisaged therapy may also benefit of medical devices and technologies currently in use for clinically approved light-based therapies (e.g., PDT, PUVA), thus facilitating its medical and regulatory acceptance. Furthermore, phototrexate could also be used as a powerful tool to study developmental processes with high spatiotemporal precision. The wavelength needed to switch phototrexate on can be scattered and absorbed by human tissue, and displays low skin penetration (below 0.3 $\mathrm{mm}) .{ }^{69}$ However, new derivatives can be developed that photoisomerize at wavelengths where penetration though body tissue is orders of magnitude better (that is, in the red to nearinfrared region). ${ }^{70,71}$ 


\section{ASSOCIATED CONTENT}

\section{Supporting Information}

The Supporting Information is available free of charge on the ACS Publications website.

Materials and methods, detailed synthetic procedures, chemical analyses and spectra, and additional data and figures as noted in the text (PDF).

\section{AUTHOR INFORMATION}

\section{Corresponding Author}

*pau@icrea.cat

\section{Author Contributions}

All authors have given approval to the final version of the manuscript. / + AGJ and NC contributed equally.

\section{Funding Sources}

This project has received funding from the European Union's Horizon 2020 Framework Programme for Research and Innovation under the Specific Grant Agreement No. 720270 (Human Brain Project SGA1), AGAUR/Generalitat de Catalunya (CERCA Programme and 2017-SGR-1442), FEDER funds, ERANET SynBio MODULIGHTOR, Fundaluce and Ramón Areces foundations, and MINECO projects CTQ2016-80066R to PG and SAF2017-83815R to CS. CM was supported by the Ermenegildo Zegna Founder's Scholarship.

\section{Notes}

The authors have filed a patent application for photoisomerizable derivatives of dihydrofolate reductase inhibitors.

\section{ACKNOWLEDGMENT}

Authors are grateful to J. M. Vela, S. Fonquerna, A. Riera, and A. Fernández-García for useful discussions. Molecular graphics and analyses were performed with the UCSF Chimera package. Chimera is developed by the Resource for Biocomputing, Visualization, and Informatics at the University of California, San Francisco (supported by NIGMS P41-GM103311). Mass spectrometry was performed at the IRB Barcelona Mass Spectrometry Core Facility, which actively participates in the BMBS European COST Action BM 1403 and is a member of Proteored, PRB2ISCIII, supported by grant PRB2 (IPT13/0001 - ISCIII-SGEFI / FEDER).

\section{REFERENCES}

65.

(1) Chabner, B. A.; Roberts, T. G. Nat Rev Cancer 2005, 5 (1),

(2) Miller, K. D.; Siegel, R. L.; Lin, C. C.; Mariotto, A. B.; Kramer, J. L.; Rowland, J. H.; Stein, K. D.; Alteri, R.; Jemal, A. CA: A Cancer Journal for Clinicians 2016, 66 (4), 271.

(3) Senapati, S.; Mahanta, A. K.; Kumar, S.; Maiti, P. Signal Transduct Target Ther 2018, 3 (1), 7 .

(4) Nicolaides, N. C.; Sass, P. M.; Grasso, L. 2010, 5 (11), 1123.

(5) Baudino, T. Curr. Drug Discov. Technol. 2015, 12 (1), 3.

(6) Sawyers, C. L. 2008, 452 (7187), 548.

(7) Saijo, N. Cancer Res. Treat. 2012, 44 (1), 1.

(8) Reeßing, F.; Szymanski, W. Curr. Med. Chem. 2017, 24 (42), 4905.
(9) Hansen, M. J.; Velema, W. A.; de Bruin, G.; Overkleeft, H. S.; Szymanski, W.; Feringa, B. L. ChemBioChem 2014, 15 (14), 2053.

(10) Szymanski, W.; Ourailidou, M. E.; Velema, W. A.; Dekker, F. J.; Feringa, B. L. Chemistry - A European Journal 2015, 21 (46), 16517.

(11) Mayer, G.; Heckel, A. Biologically active molecules with a "light switch".; 2006; Vol. 45, pp 4900-4921.

(12) Riggsbee, C. W.; Deiters, A. Trends Biotechnol. 2010, 28 (9), 468.

(13) Beharry, A. A.; Woolley, G. A. Chem Soc Rev 2011, 40 (8), 4422

(14) Brieke, C.; Rohrbach, F.; Gottschalk, A.; Mayer, G.; Heckel, A. Angew. Chem. Int. Ed. Engl. 2012, 51 (34), 8446.

(15) Szymanski, W.; Beierle, J. M.; Kistemaker, H. A. V.; Velema, W. A.; Feringa, B. L. Chem. Rev. 2013, 113 (8), 6114.

(16) Agostinis, P.; Berg, K.; Cengel, K. A.; Foster, T. H.; Girotti, A. W.; Gollnick, S. O.; Hahn, S. M.; Hamblin, M. R.; Juzeniene, A.; Kessel, D.; Korbelik, M.; Moan, J.; Mroz, P.; Nowis, D.; Piette, J.; Wilson, B. C.; Golab, J. 2011, 61 (4), 250.

(17) See, K. L.; Forbes, I. J.; Betts, W. H. Photochem Photobiol 1984, $39(5), 631$.

(18) Mitchell, J. B.; McPherson, S.; DeGraff, W.; Gamson, J.; Zabell, A.; Russo, A. Cancer Res. 1985, 45 (5), 2008.

(19) Busch, T. M.; Wileyto, E. P.; Emanuele, M. J.; Del Piero, F.; Marconato, L.; Glatstein, E.; Koch, C. J. Cancer Res. 2002, 62 (24), 7273 .

(20) Fink, C.; Uhlmann, L.; Enk, A.; Gholam, P. J Eur Acad Dermatol Venereol 2016, 31 (1), 70.

(21) Babes, A.; Sauer, S. K.; Moparthi, L.; Kichko, T. I.; Neacsu, C.; Namer, B.; Filipovic, M.; Zygmunt, P. M.; Reeh, P. W.; Fischer, M. J. M. J. Neurosci. 2016, 36 (19), 5264.

(22) Zhang, P.; Sadler, P. J. European Journal of Inorganic Chemistry 2017, 2017 (12), 1541.

(23) Ciesienski, K. L.; Hyman, L. M.; Yang, D. T.; Haas, K. L.; Dickens, M. G.; Holbrook, R. J.; Franz, K. J. European Journal of Inorganic Chemistry 2010, 2010 (15), 2224.

(24) Noguchi, M.; Skwarczynski, M.; Prakash, H.; Hirota, S.; Kimura, T.; Hayashi, Y.; Kiso, Y. Bioorganic \& Medicinal Chemistry 2008, 16 (10), 5389

(25) Bio, M.; Rajaputra, P.; Nkepang, G.; Awuah, S. G.; Hossion, A. M. L.; You, Y. J. Med. Chem. 2013, 56 (10), 3936.

(26) Velema, W. A.; Szymanski, W.; Feringa, B. L. J. Am. Chem. Soc. 2014, 136 (6), 2178.

(27) Broichhagen, J.; Frank, J. A.; Trauner, D. Acc. Chem. Res. 2015, 48 (7), 1947.

(28) Lerch, M. M.; Hansen, M. J.; van Dam, G. M.; Szymanski, W.; Feringa, B. L. Angew. Chem. Int. Ed. Engl. 2016, 55 (37), 10978.

(29) Ankenbruck, N.; Courtney, T.; Naro, Y.; Deiters, A. Angew. Chem. Int. Ed. Engl. 2018, 57 (11), 2768.

(30) Pittolo, S.; Gómez-Santacana, X.; Eckelt, K.; Rovira, X.; Dalton, J.; Goudet, C.; Pin, J.-P.; Llobet, A.; Giraldo, J.; Llebaria, A.; Gorostiza, P. Nat. Chem. Biol. 2014, 10 (10), 813.

(31) Broichhagen, J.; Johnston, N. R.; Ohlen, von, Y.; MeyerBerg, H.; Jones, B. J.; Bloom, S. R.; Rutter, G. A.; Trauner, D.; Hodson, D. J. Angew. Chem. Int. Ed. Engl. 2016, 55 (19), 5865.

(32) Agnetta, L.; Kauk, M.; Canizal, M. C. A.; Messerer, R.; Holzgrabe, U.; Hoffmann, C.; Decker, M. Angew. Chem. Int. Ed. Engl. 2017, 56 (25), 7282.

(33) Bregestovski, P.; Maleeva, G.; Gorostiza, P. Br. J. Pharmacol. 2017, 172 (1), 5870.

(34) Wegener, M.; Hansen, M. J.; Driessen, A. J. M.; Szymanski, W.; Feringa, B. L. J. Am. Chem. Soc. 2017, 139 (49), 17979.

(35) Nevola, L.; Martín-Quirós, A.; Eckelt, K.; Camarero, N.; Tosi, S.; Llobet, A.; Giralt, E.; Gorostiza, P. Angew. Chem. Int. Ed. Engl. 2013, 52 (30), 7704.

(36) Presa, A.; Brissos, R. F.; Caballero, A. B.; Borilovic, I.; Korrodi-Gregório, L.; Pérez-Tomás, R.; Roubeau, O.; Gamez, P. Angew. Chem. Int. Ed. Engl. 2015, 54 (15), 4561.

(37) Babii, O.; Afonin, S.; Garmanchuk, L. V.; Nikulina, V. V.; Nikolaienko, T. V.; Storozhuk, O. V.; Shelest, D. V.; Dasyukevich, O. 
I.; Ostapchenko, L. I.; Iurchenko, V.; Zozulya, S.; Ulrich, A. S.; Komarov, I. V. Angew. Chem. 2016, 128 (18), 5583.

(38) Borowiak, M.; Nahaboo, W.; Reynders, M.; Nekolla, K.; Jalinot, P.; Hasserodt, J.; Rehberg, M.; Delattre, M.; Zahler, S.; Vollmar, A.; Trauner, D.; Thorn-Seshold, O. Cell 2015, 162 (2), 403.

(39) Engdahl, A. J.; Torres, E. A.; Lock, S. E.; Engdahl, T. B.; Mertz, P. S.; Streu, C. N. Org. Lett. 2015, 17 (18), 4546.

(40) Sheldon, J. E.; Dcona, M. M.; Lyons, C. E.; Hackett, J. C.; Hartman, M. C. T. Org. Biomol. Chem. 2016, 14 (1), 40.

(41) Rastogi, S. K.; Zhao, Z.; Barrett, S. L.; Shelton, S. D.; Zafferani, M.; Anderson, H. E.; Blumenthal, M. O.; Jones, L. R.; Wang, L.; Li, X.; Streu, C. N.; Du, L.; Brittain, W. J. Eur J Med Chem 2018, 143,1 .

(42) Enna, S. J.; Bylund, D. B. xPharm: the comprehensive pharmacology reference; 2008.

(43) Hamblin, M. R.; Huang, Y.-Y. Handbook of Photomedicine; CRC Press, 2013.

(44) https://www.drugbank.ca/.

(45) Gaulton, A.; Hersey, A.; Nowotka, M.; Bento, A. P.; Chambers, J.; Mendez, D.; Mutowo, P.; Atkinson, F.; Bellis, L. J.; Cibrián-Uhalte, E.; Davies, M.; Dedman, N.; Karlsson, A.; Magariños, M. P.; Overington, J. P.; Papadatos, G.; Smit, I.; Leach, A. R. Nucleic Acids Res. 2017, 45 (D1), D945.

(46) Berman, H. M.; Westbrook, J.; Feng, Z.; Gilliland, G.; Bhat, T. N.; Weissig, H.; Shindyalov, I. N.; Bourne, P. E. Nucleic Acids Res. 2000, 28 (1), 235.

(47) Prescription Drug Information, Interactions \& Side Effects https://www.drugs.com/.

(48) Vander Heiden, M. G. Nature Reviews Drug Discovery 2011, 10 (9), 671.

(49) Luengo, A.; Gui, D. Y.; Vander Heiden, M. G. Cell Chem Biol 2017, 24 (9), 1161.

(50) Kanarek, N.; Keys, H. R.; Cantor, J. R.; Lewis, C. A.; Chan, S. H.; Kunchok, T.; Abu-Remaileh, M.; Freinkman, E.; Schweitzer, L. D.; Sabatini, D. M. Nature 2018, 559 (7715), 632.

(51) Methotrexate https://www.drugbank.ca/drugs/DB00563.

(52) Castillo, V. S.; Moyano, L. A. Methotrexate: Pharmacology, clinical uses and adverse effects.; Nova Biomedical.

(53) Bartels, E.; Wassermann, N. H.; Erlanger, B. F. Proc. Natl. Acad. Sci. U.S.A. 1971, 68 (8), 1820.

(54) Volgraf, M.; Gorostiza, P.; Szobota, S.; Helix, M. R.; Isacoff, E. Y.; Trauner, D. J. Am. Chem. Soc. 2007, 129 (2), 260.
(55) Huckvale, R.; Mortensen, M.; Pryde, D.; Smart, T. G.; Baker, J. R. Org. Biomol. Chem. 2016, 14 (28), 6676.

(56) Schoenberger, M.; Damijonaitis, A.; Zhang, Z.; Nagel, D.; Trauner, D. ACS Chem Neurosci 2014, 5 (7), 514.

(57) Gómez-Santacana, X.; Pittolo, S.; Rovira, X.; Lopez, M.; Zussy, C.; Dalton, J. A. R.; Faucherre, A.; Jopling, C.; Pin, J.-P.; Ciruela, F.; Goudet, C.; Giraldo, J.; Gorostiza, P.; Llebaria, A. ACS Cent Sci 2017, 3 (1), 81.

(58) Cody, V.; Luft, J. R.; Pangborn, W.; IUCr. Acta Crystallogr Sect D Biol Crystallogr 2005, 61 (2), 147.

(59) DeGraw, J. I.; Kisliuk R. L.; Gaumont Y.; Baugh C. M.; Nair M. G. J. Med. Chem. 1974, 17 (5), 552.

(60) Sirotnak, F. M.; DeGraw, J. I.; Moccio, D. M.; Samuels, L. L.; Goutas, L. J. Cancer Chemother. Pharmacol. 1984, 12 (1), 18.

(61) Hynes, J. B.; Harmon, S. J.; Floyd, G. G.; Farrington, M.; Hart, L. D.; Gale, G. R.; Washtien, W. L.; Susten, S. S.; Freisheim, J. H. J. Med. Chem. 1985, 28 (2), 209.

(62) Hynes, J. B.; Patil, S. A.; Tomazic, A.; Kumar, A.; Pathak, A.; Tan, X.; Li, X.; Ratnam, M.; Delcamp, T. J.; Freisheim, J. H. J. Med. Chem. 1988, 31 (2), 449.

(63) Toledo-Sherman, L. M.; Desouza, L.; Hosfield, C. M.; Liao, L.; Boutillier, K.; Taylor, P.; Climie, S.; McBroom-Cerajewski, L.; Moran, M. F. Clinical Proteomics 2004, 1 (1), 45.

(64) Glauner, H.; Ruttekolk, I. R.; Hansen, K.; Steemers, B.; Chung, Y.-D.; Becker, F.; Hannus, S.; Brock, R. Br. J. Pharmacol. 2010, 160 (4), 958.

(65) Eichholtz, H.; Trott, K. R. Br. J. Cancer 1980, 41 (2), 277.

(66) Copeland, R. A. Evaluation of Enzyme Inhibitors in Drug Discovery; John Wiley \& Sons: Hoboken, NJ, USA, 2013.

(67) Lee, M. S.; Bonner, J. R.; Bernard, D. J.; Sanchez, E. L.; Sause, E. T.; Prentice, R. R.; Burgess, S. M.; Brody, L. C. BMC Developmental Biology 2012 12:1 2012, 12 (1), 12.

(68) Kimmel, C. B.; Ballard, W. W.; Kimmel, S. R.; Ullmann, B.; Schilling, T. F. Dev. Dyn. 1995, 203 (3), 253.

(69) Avci, P.; Gupta, A.; Sadasivam, M.; Vecchio, D.; Pam, Z.; Pam, N.; Hamblin, M. R. Semin Cutan Med Surg 2013, 32 (1), 41.

(70) Cheong, W. F.; Prahl, S. A.; Welch, A. J. IEEE Journal of Quantum Electronics 1990, 26 (12), 2166.

(71) Dong, M.; Babalhavaeji, A.; Samanta, S.; Beharry, A. A.; Woolley, G. A. Acc. Chem. Res. 2015, 48 (10), 2662. 
Table of Contents Graphic

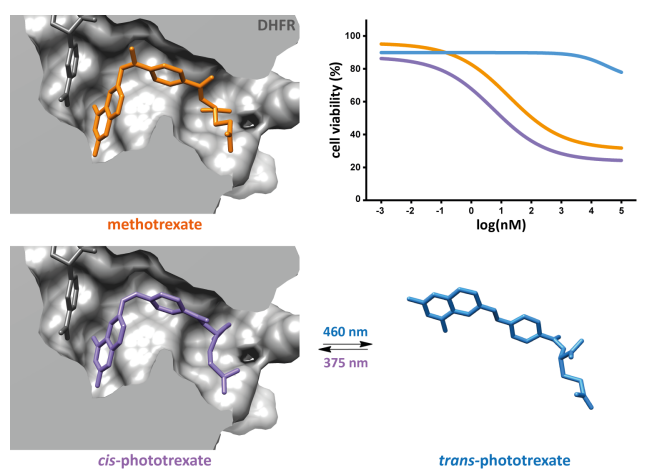

